\title{
Exploring short period oscillations in filaments
}

\author{
D. Y. Kolobov N. I. Kobanov and S. A. Chupin \\ Institute of Solar-Terrestrial Physics, P.O. Box 291, 664033, Irkutsk, Russia \\ email: kolobov@iszf.irk.ru
}

\begin{abstract}
The authors analyse sources of false Doppler velocity signals of high frequencies $(10 \mathrm{mHz}$ and higher) in observations of filaments. In ground-based observations, spectrograph noise and image shifting at the spectrograph entrance slit are the main causes of the false signal. It is shown that using differential methods and telluric lines as reference lines significantly reduces the influence of the first factor. Periodical image shifting along the spectrograph slit can be compensated for during data reduction. In some cases detected high-frequency oscillations appear to be real.
\end{abstract}

Keywords. Sun: filaments, Sun: oscillations, Sun: chromosphere

\section{Introduction}

An interest in oscillations in filaments has existed for a long time (Malherbe, Schmieder \& Mein (1981), Bashkirtsev, Kobanov \& Mashnich (1983), Tsubaki \& Takeuchi (1986)). However, reliable observational evidence of high-frequency oscillations has been reported only once by Balthasar, et al. (1993). Obviously, this situation is due to a set of instrumental problems. Firstly, spurious oscillations can be caused by oscillations in the Earth's atmosphere. They can produce brightness variations, focus changing and jittering, and these effects are modulated by telescope drifts. Spectrograph noise can also play an important role. An image acquisition system can induce camera noise and frequency aliasing and it is supposed to work at the edge of its capabilities. Even if someone were to overcome the above problems and were to detect high-frequency oscillations, the question of whether these oscillations belong to a filament or to the chromosphere would remain.

The data under investigation were obtained during the 2007 observational season. It contains 20 time series. A spectropolarimeter allows one to get simultaneously line-ofsight (LOS) velocity signal in $\mathrm{H} \alpha$, telluric line shifts, and velocity signal in the photospheric line Fe I $6569 \AA, g=1.4$ (in some time series a signal of the magnetic field is available).

\section{Test of the origins of observed high frequency oscillations}

The test for the above mentioned artefacts is described here. The image acquisition system must provide high temporal resolution (down to $0.25 \mathrm{~s}$ ) and exact synchronisation of frame grabbing. Flat field correction is also necessary. Figure 1 presents the situation when the system does not provide exact synchronisation, and the system gets a set of frames with different exposure times. One can see a high frequency peak at $325 \mathrm{mHz}$ in the intensity signal. Note the absence of this peak in the velocity signal. This is because of the method used - velocity is obtained with the so-called "Lambda meter" mode.

Figure 2 shows a power spectra of a time series made in filament ( $\mathrm{H} \alpha$-line). An equidistant spectrum with maxima spaced by $45 \mathrm{mHz}(47,92,137,182 \mathrm{mHz})$ is clearly seen. 

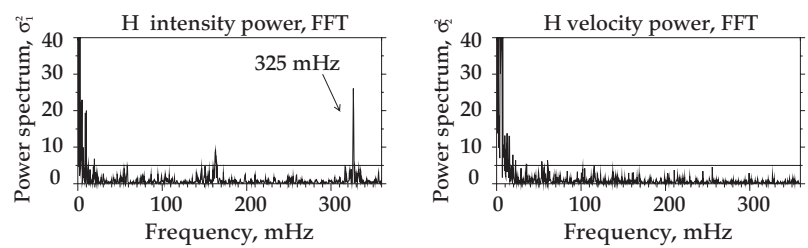

Figure 1. Spurius high frequency signal. FFT power spectra: $\mathrm{H} \alpha$ intensity (left panel); $\mathrm{H} \alpha$ LOS velocity, "lambda meter mode" (right panel). Time series 2007 June 26
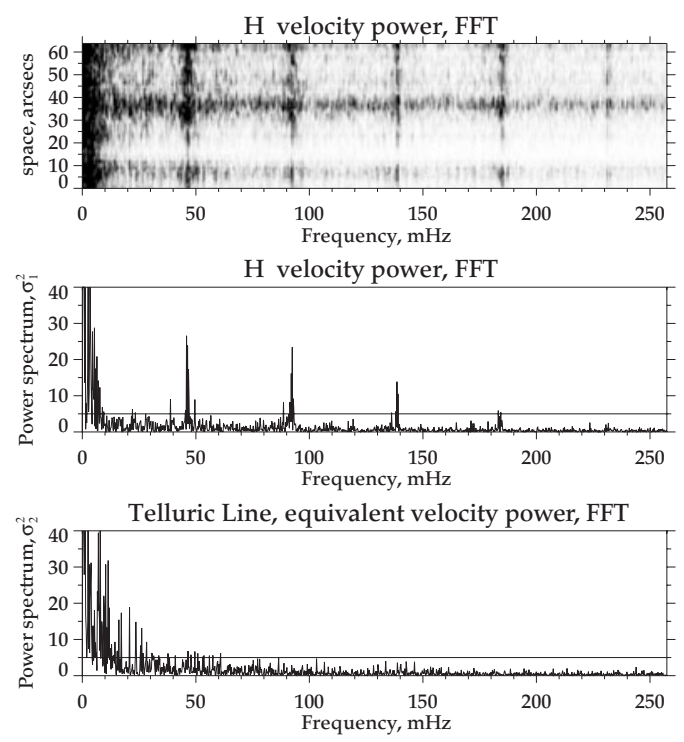

Figure 2. High frequency oscillations, produced by the Earth's atmosphere and telescope's guiding system. FFT power spectra: $\mathrm{H} \alpha$ LOS velocity power for all spatial points along the slit (upper panel); $\mathrm{H} \alpha$ LOS velocity power, single slit position (middle panel); telluric line, single slit position (bottom panel). Time series 2007 July 17, cadence $0.5 \mathrm{~s}$

In the telluric power spectrum high-frequency peaks are absent, which excludes spectrograph noise as their source (Fig. 2c). Note the spatial distribution of high-frequency peaks - they are registered almost in every point along the slit. The power distribution cannot be caused by aliasing: 5 time series with different cadence show exactly the same distribution of peaks. Apparently this picture is produced by atmospheric oscillations probably modulated by the guiding system that finally leads to the image jittering on the spectrograph slit.

Another candidate for the production high-frequency spurious oscillations is the spectrograph. Basic methods to suppress its influence are the use of telluric lines and differential measurements of LOS velocity in two spatial points on the Sun (Kobanov (1983)). Usually spectrograph slit is pointed to a single position on the solar disk. But it is possible to make an optical scheme which could switch the slit position between two spatial points (figure $3(\mathrm{a}))$. At first, the slit is in the first position, then in the second $(\Delta t=0.5 \mathrm{~s})$ position. Finally we get two time series in different points of the filament. The resulting series, without spectrograph noise, is obtained by subtracting one series from the other. The observations carried out with this technique did not reveal any oscillations with a frequency above $10 \mathrm{mHz}$, but we were able to detect travelling waves near the filament's boundary, which is shown on the wavelet filtered (see Torrence \& Compo (1998) for 

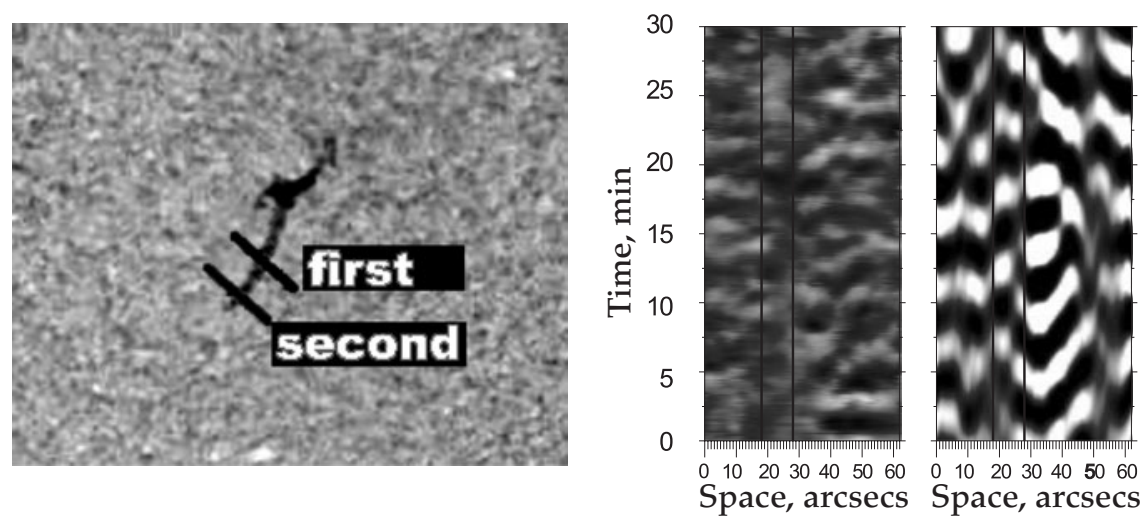

Figure 3. Differential method to suppress spectrograph noise: first and second slit positions (left panel); space-time diagram of LOS velocity of ordinary time-series (middle panel); time-series obtained with the help of the differential method, space-time LOS velocity diagram filtered with wavelet for $3 \mathrm{mHz}$ (right panel). Vertical lines mark the filament's boundaries (time series 2007 July 21 , cadence $0.5 \mathrm{~s}$ )

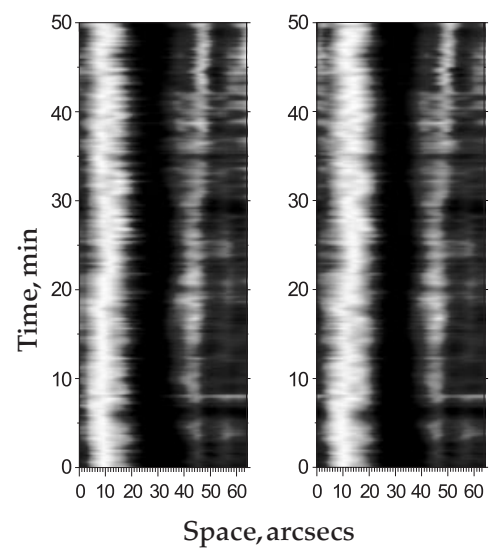

Figure 4. Space-time $\mathrm{H} \alpha$ intensity diagram: initial time-series (left panel); series after compensation for image displacement along the spectrograph slit by means of cross-wavelet (right panel). Time series 2007 July 18 , cadence $0.25 \mathrm{~s}$

details) space-time diagram of the LOS velocity (see figure 3(c)). Such a picture can be regarded as evidence of solar origin of these oscillations. We could not find any artefact which could produce it.

In searching for high frequency oscillations, the problem of object displacements on the slit must be solved. Those displacements can have different nature. Firstly, it is a jittering along the slit. Although the guide system compensates slow movements, high frequency movements cannot be compensated for. It is a well known fact that filaments have proper motions. They cannot be compensated for by the guide as it tracks the solar disk as a whole. What ways are there to compensate for those displacements? The following solutions are possible. A filament is visible in $\mathrm{H} \alpha$-core brightness, so it is possible to correct its position by: 1) with the intensity profile minimum; 2) cross correlation with previous frames (usually this procedure is applied locally and needs step-by-step assistance); 3) with cross-wavelet transformation. The last yields a tool to distinguish fine structure, profile shape and allows for tuning between fine structure and average profile. All these methods demand flat-field correction. 

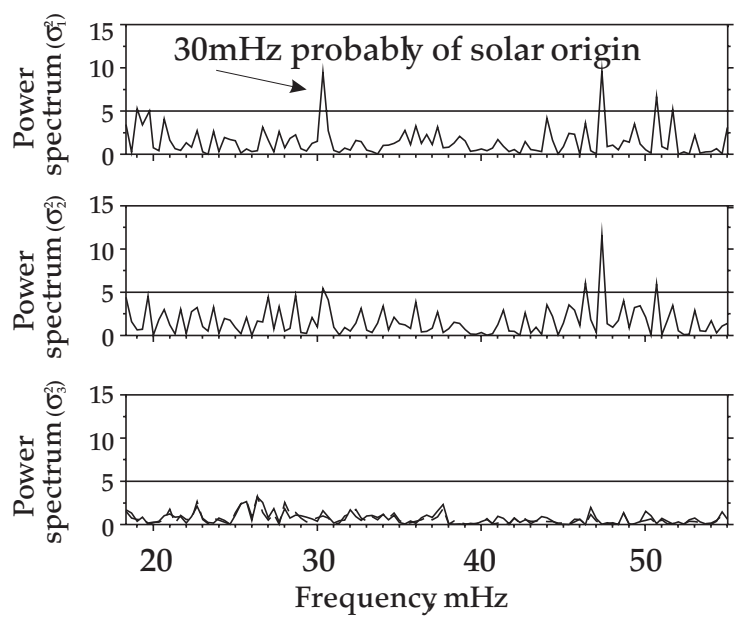

Figure 5. FFT power of $\mathrm{H} \alpha$ LOS velocity: series after compensation for image displacement along the spectrograph slit by means of cross-wavelet (upper panel); initial time-series (middle panel); FFT power of telluric line shifts before (dashed line) and after (solid line) alignment (bottom panel). Time series 2007 July 18, cadence $0.25 \mathrm{~s}$, length $50 \mathrm{~min}$

Figure 4 demonstrates time series in the filament (intensity signal) before (figure 4(b)) and after (figure 4(c)) alignment with the help of cross-wavelet. The corresponding FFT power spectrum for this time series is shown in figure 5 . The aligned time series shows a significant peak of $30 \mathrm{mHz}$. The horizontal line marks the $99 \%$ confidence level. Time series without alignment do not show this peak. The telluric line does not show this peak neither with alignment nor without it, therefore spectrograph noise cannot be the source of these oscillations. Spatially these oscillations are confined by the filament's boundaries. Probably these oscillations are of solar origin.

\section{Conclusions}

Atmospheric oscillations are the most probable source of spurious oscillations. Propagating waves can serve as a diagnostic tool to confirm the solar origin of detected oscillations. A significant frequency peak of $30 \mathrm{mHz}$ was found in one filament, and is very likely to be of solar origin. These oscillations are probably distributed over small spatial scales. Further research is needed to clarify whether this kind of oscillation is connected with filaments or the chromosphere.

\section{Acknowledgements}

The work is supported by the RFBR grant 05-02-16325 and RAS program No. 16.

\section{References}

Balthasar H., Wiehr E., Schleicher H. \& Wöhl H. 1993, A\& A 277, 645

Bashkirtsev V.S., Kobanov N.I. \& Mashnich G.P. 1983, Sol. Phys. 82, 443

Kobanov N.I. 1983, Sol. Phys. 82, 237

Malherbe J. M.,; Schmieder B. \& Mein P. 1981, A\& A 102, 124

Tsubaki, T. \& Takeuchi, A. 1986, Sol. Phys. 104, 313

Torrence C. \& Compo G.P. 1998, Bull. Am. Meteo. Soc. 79, 61 